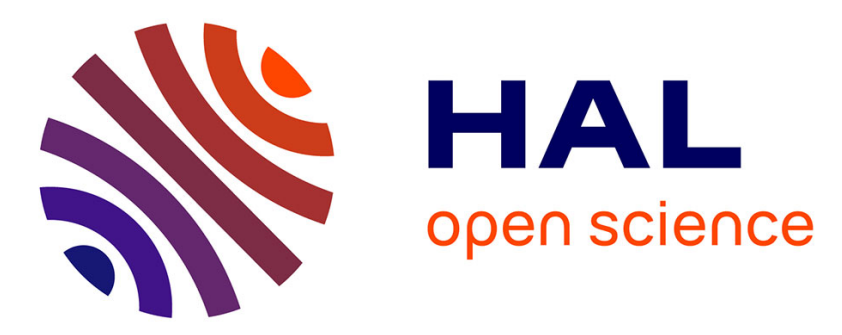

\title{
Étude par spectrométrie Mössbauer de ferrites de lithium substitués. Influence des champs hyperfins supertransferrés et de la relaxation
}

\author{
J.L. Dormann
}

\section{- To cite this version:}

J.L. Dormann. Étude par spectrométrie Mössbauer de ferrites de lithium substitués. Influence des champs hyperfins supertransferrés et de la relaxation. Revue de Physique Appliquée, 1980, 15 (6), pp.1113-1121. 10.1051/rphysap:019800015060111300 . jpa-00244829

HAL Id: jpa-00244829

https://hal.science/jpa-00244829

Submitted on 1 Jan 1980

HAL is a multi-disciplinary open access archive for the deposit and dissemination of scientific research documents, whether they are published or not. The documents may come from teaching and research institutions in France or abroad, or from public or private research centers.
L'archive ouverte pluridisciplinaire HAL, est destinée au dépôt et à la diffusion de documents scientifiques de niveau recherche, publiés ou non, émanant des établissements d'enseignement et de recherche français ou étrangers, des laboratoires publics ou privés. 


\title{
Étude par spectrométrie Mössbauer de ferrites de lithium substitués. Influence des champs hyperfins supertransferrés et de la relaxation
}

\author{
J. L. Dormann \\ Laboratoire de Magnétisme, C.N.R.S., 1, place A.-Briand, 92190 Meudon-Bellevue, France
}

(Reçu le 16 novembre 1979, révisé le 11 janvier 1980, accepté le 15 janvier 1980)

\begin{abstract}
Résumé. - Des séries de ferrites de Li substitués avec du zinc, du titane et du chrome ont été étudiées de 4 à $300 \mathrm{~K}$. Les paramètres hyperfins des différents sites élémentaires de fer ont été déterminés. La variation du champ hyperfin supertransferré $\Delta H_{\mathrm{HST}}$ due à la substitution de $\mathrm{Zn}$ ou de $\mathrm{Li}$ en site $\mathrm{A}$, de $\mathrm{Ti}$, de $\mathrm{Cr}$ ou de $\mathrm{Li}$ en site $\mathrm{B}$ a été établie, ainsi que la variation du champ hyperfin du $\mathrm{Fe}_{\mathrm{B}}$ due à la substitution de $\mathrm{Cr}$. Une valeur de $\Delta H_{\mathrm{HST}}(\mathrm{A})$ plus grande que les valeurs habituelles est trouvée pour la substitution de $\mathrm{Ti}$. Les phénomènes de relaxation usuellement observés dans les ferrites substitués avec du $\mathrm{Zn}$ ont été observés pour les ferrites de $\mathrm{Li}-\mathrm{Zn}$, mais aussi pour les ferrites de Li-Ti à des taux de substitution et températures plus élevés. Ces phénomènes sont discutés en relation avec la structure non colinéaire des spins des $\mathrm{Fe}_{\mathrm{B}}$ (ferrites de $\mathrm{Li}-\mathrm{Zn}$ ) ou avec celle prévisible des spins des $\mathrm{Fe}_{\mathrm{A}}$ (ferrites de Li-Ti).
\end{abstract}

\begin{abstract}
Several substituted lithium ferrites (with $\mathrm{Zn}, \mathrm{Ti}$ and $\mathrm{Cr}$ ) have been studied between 4 and $300 \mathrm{~K}$. The hyperfine parameters of the different sites are deduced. The variation of the supertransferred hyperfine field $\Delta H_{\text {STHF }}$ produced by the $\mathrm{Zn}$ and $\mathrm{Li}$ substitution to the $\mathrm{A}$ site $\mathrm{Fe}$ and that of the $\mathrm{Ti}, \mathrm{Li}$ and $\mathrm{Cr}$ to the $\mathrm{B}$ site $\mathrm{Fe}$ is given. The variation of the hyperfine field produced by the $\mathrm{Cr}$ substitution to the $\mathrm{B}$ site $\mathrm{Fe}$ is also given. For $\mathrm{Ti}$ substitution the value of $\Delta H_{\mathrm{STHF}}(\mathrm{A})$ is found higher than usual values. The same relaxation phenomena as in the case of substituted $\mathrm{Zn}$ ferrites are observed in $\mathrm{Li}-\mathrm{Zn}$ ferrites. The same phenomenum is also observed in $\mathrm{Li}-\mathrm{Ti}$ ferrites but corresponding to higher substitution and higher temperature. These relaxation phenomena are related to the localized canting of the B site Fe spins existing in $\mathrm{Li}-\mathrm{Zn}$ ferrites and to the supposed localized canting of the A site $\mathrm{Fe}$ spins in the Li-Ti ferrites.
\end{abstract}

Le ferrite de lithium présente des propriétés magnétiques et électriques très intéressantes pour son utilisation dans des dispositifs, notamment en hyperfréquence. De nombreuses substitutions ont été étudiées en vue d'améliorer ses caractéristiques, en particulier par le zinc et le titane. La majeure partie des propriétés magnétiques de ces ferrites de lithium substitués est bien établie, mais certains points sont encore discutés, en particulier la structure magnétique du ferrite de Li-Zn.

Les ferrites cristallisent dans la structure spinelle. Celle-ci correspond à un empilement compact (cubique à face centrée) d'anions oxygène délimitant deux types de sites où se placent les cations métalliques. Les sites octaédriques (B) occupés sont deux fois plus nombreux que les sites tétraédriques (A) occupés. $\mathrm{La}$ maille élémentaire comprend huit fois le motif $\mathrm{AB}_{2} \mathrm{O}_{4}$. Pour le $\mathrm{Fe}^{3+}$, les interactions d'échange A-A, $\mathrm{A}-\mathrm{B}$ et $\mathrm{B}-\mathrm{B}$ sont antiferromagnétiques. L'interaction A-B étant prédominante, l'ordre magnétique résul- tant est celui de deux sous-réseaux ferromagnétiques couplés antiferromagnétiquement.

La distribution des cations [1] dans le ferrite de Li-Ti s'écrit :

$$
\left[\mathrm{Fe}_{1-0,5 t}^{3+} \mathrm{Li}_{0,5 t}^{1+}\right]_{\mathrm{A}}\left[\mathrm{Fe}_{1,5-t}^{3+} \mathrm{Li}_{0,5}^{1+} \mathrm{Ti}_{t}^{4+}\right]_{\mathrm{B}} \mathrm{O}_{4} .
$$

Le moment magnétique à $0 \mathrm{~K}$ et la température de Curie décroissent, la maille cristalline croît lorsque la teneur en titane augmente [1]; pour $T \lesssim 0,7, M=f(t)$ suit une loi de dilution simple, mais avec une pente légèrement supérieure à celle déduite de la distribution (1).

Déduite des mesures d'aimantation [2,3] des ferrites de $\mathrm{Li}-\mathrm{Zn}$ ayant des taux de substitution inférieurs à 0,25 , la distribution des cations s'écrit :

$$
\left[\mathrm{Fe}_{1-z}^{3+} \mathrm{Zn}_{z}^{2+}\right]_{\mathrm{A}}\left[\mathrm{Fe}_{1,5+0,5_{z}}^{3+} \mathrm{Li}_{0,5-0,5_{z}}^{1+}\right]_{\mathrm{B}} \mathrm{O}_{4} .
$$

L'addition de zinc devrait avoir pour effet l'augmentation du moment magnétique total, mais pour 
$z>0,3$, il est observé une diminution du moment magnétique par rapport à une loi de dilution simple. Plusieurs raisons ont été évoquées pour expliquer cette propriété : (i) la distribution des cations est différente de (2); (ii) certains ou la totalité des spins des $\mathrm{Fe}_{\mathrm{B}}$ ne sont ni alignés entre eux, ni alignés avec les spins des $\mathrm{Fe}_{\mathrm{A}}[4,5]$ diminuant ainsi le moment magnétique total; (iii) certains des $\mathrm{Fe}_{\mathrm{B}}$ sont dans un état paramagnétique $[6,7,8]$ et il peut $y$ avoir formation de grains ferromagnétiques.

Plusieurs travaux récents permettent d'élucider ce problème. D'une part, des mesures de diffraction de rayons $X$ et de neutrons [9] ont confirmé la distribution (2). D'autre part, Zhilyakov et al. [10] à partir de mesures de diffraction de neutrons ont confirmé l'existence d'une structure non colinéaire des spins des $\mathrm{Fe}_{\mathbf{B}}$ différente d'une structure Yafet-Kittel [4] et ont interprété leurs mesures à l'aide du localized canting Model (LCM) de Rosencwaig [11]. Enfin White et al. [12] ont mesuré l'aimantation sous champ intense et ont obtenu un bon accord qualitatif avec le modèle de Rosencwaig.

Dans ce modèle, un champ moléculaire local $\mathbf{H}$ dépendant des spins des premiers voisins est écrit. Par exemple, pour un atome de fer en site B :

$$
\mathbf{H}_{\mathbf{B}}=\frac{2}{g \mu_{\mathrm{B}}}\left[\sum_{\mathrm{B}} J_{\mathrm{BB}} \mathbf{S}_{\mathbf{B}}+\sum_{\mathrm{A}} J_{\mathrm{AB}} \mathbf{S}_{\mathrm{A}}\right] .
$$

La sommation est faite sur les premiers voisins $\mathrm{A}$ et $\mathrm{B}, J_{\mathrm{AB}}$ et $J_{\mathrm{BB}}$ représentent les intégrales d'échange.

$\mathrm{Si}$ des atomes diamagnétiques sont substitués en sites $\mathrm{A}$ et $\mathrm{B}, \mathbf{H}_{\mathrm{B}}$ dépend du nombre total $n_{\mathrm{A}}$ et $n_{\mathrm{B}}$ de premiers voisins $A$ et $B$, et du nombre de cations diamagnétiques $m$ et $q$ premiers voisins respectivement en site A et B. Si la substitution a lieu principalement en site $A$ et qu'il existe une structure non colinéaire, l'état d'énergie minimum est obtenu lorsque les spins $A$ sont parallèles entre eux et que $S_{B}$ fait un angle local $\theta$ avec $S_{\mathrm{A}}$ donné par :

$$
\theta=\frac{1-C \cos \bar{\theta}}{\left[1+C^{2}-2 C \cos \bar{\theta}\right]^{1 / 2}}
$$

où $C=\frac{n_{\mathrm{B}}-q}{n_{\mathrm{A}}-m} \frac{J_{\mathrm{BB}}}{J_{\mathrm{AA}}}$ et $\bar{\theta}$ est l'angle moyen entre $\mathbf{S}_{\mathrm{A}}$ et $\mathbf{S}_{\mathbf{B}}$.

L'angle $\bar{\theta}$ pouvant être calculé à partir des probabilités de trouver $m$ et $q$ premiers voisins diamagnétiques, le système ne dépend que d'un paramètre $J_{\mathrm{BB}} / J_{\mathrm{AA}}$. L'angle $\theta$ varie donc localement alors que dans la structure Yafet-Kittel il est constant.
Un très bon exposé des propriétés magnétiques des ferrites de Li substitués avec du $\mathrm{Zn}$, du Ti et du $\mathrm{Zn}$ et Ti est fait dans la référence [13].

Les ferrites de $\mathrm{Li}-\mathrm{Ti}$ et de $\mathrm{Li}-\mathrm{Zn}$ n'ont pas été beaucoup étudiés par spectrométrie Mössbauer. Young et al. [14] ont interprété leurs mesures à partir de l'hypothèse (iii) mais leurs spectres calculés ne correspondent pas bien aux spectres expérimentaux. Abedilo et al. [15] ont tenté de mettre en évidence la structure non colinéaire, mais la composition étudiée n'était pas assez riche en zinc. Par contre, les ferrites de $\mathrm{Ni}-\mathrm{Zn}$ et de $\mathrm{Co}-\mathrm{Zn}$ ont fait l'objet de nombreuses études. D'une part, les auteurs ont mis en évidence la structure non colinéaire pàr des expériences de spectrométrie Mössbauer sous champ appliqué (de 15 à $70 \mathrm{kOe}$ ) analysées à partir du modèle de YafetKittel [16-18] ou du L.C.M. [19, 20]; d'autre part, d'autres auteurs se sont attachés à expliquer l'évolution anormale des spectres en fonction de la température et à l'analyser en terme de relaxation [16, 21 , 22]. Tous les auteurs n'ont considéré qu'un seul champ hyperfin pour le $\mathrm{Fe}_{\mathrm{B}}$ avec un sextuplet élargi, négligeant la variation du champ hyperfin supertransferré $H_{\mathrm{HST}}$ due à la présence d'atomes non magnétiques [23] en site $\mathrm{A}$.

Pour apporter des renseignements supplémentaires sur les $H_{\mathrm{HST}}$ et les phénomènes de relaxation, nous avons étudié par spectrométrie Mossbauer plusieurs séries de ferrites de lithium substitués avec du $\mathrm{Zn}$ (non magnétique allant en site $\mathrm{A}$ ), du Ti (non magnétique allant en site $\mathrm{B}$ ) et du $\mathrm{Cr}$ (magnétique allant en site $B$ ).

1. Résultats expérimentaux. - Les ferrites de lithium substitués étudiés se groupent en quatre séries : les ferrites de Li-Ti substitués avec du titane (formule (1)), les ferrites de Li-Zn substitués avec du zinc (formule (2)), les ferrites de Li-Zn-Ti substitués avec du zinc et du titane (formule (3)) et les ferrites de Li-Cr substitués avec du chrome (formule (4)).

$$
\begin{gathered}
{\left[\mathrm{Fe}_{1-z}^{3+} \mathrm{Zn}_{z}^{2+}\right]_{\mathrm{A}}\left[\mathrm{Fe}_{1,5+0.5(z-3 t)}^{3+} \mathrm{Li}_{0.5+0.5(t-z)}^{1+} \mathrm{Ti}_{t}^{4+}\right]_{\mathrm{B}} \mathrm{O}_{4}} \\
(t \leqslant z) \\
{\left[\mathrm{Fe}_{1}^{3+}\right]_{\mathrm{A}}\left[\mathrm{Fe}_{1,5-x}^{3+} \mathrm{Li}_{0,5}^{1+} \mathrm{Cr}_{x}\right]_{\mathrm{B}} \mathrm{O}_{4}}
\end{gathered}
$$

Les taux de substitutions étudiés pour les 3 premières séries sont indiqués dans le tableau $\mathrm{I}$ et pour les ferrites de $\mathrm{Li}-\mathrm{Cr}$ sont $x=0,0,133$ et 0,4 .

Les échantillons des 3 premières séries sont polycristallins et ont été préparés à partir de poudres d'oxydes, puis broyés, chamottés et enfin frittés en

Tableau I. - Taux de substitutions étudiées : zinc (z), titane $(t)$ et manganèse $(m)$.

$\begin{array}{ccccccccc}z & 0 & 0,25 & 0,4 & 0,6 & 0 & 0 & 0 & 0 \\ - & \overline{0} & \overline{-} & \overline{-} & \overline{-} & - & - & - & - \\ t & 0 & 0 \text { et } 0,15 & 0 \text { et } 0,15 & 0,15 & 0,3 & 0,56 & 0,735 \\ m & 0 \text { et } 0,035 & 0 \text { et } 0,035 & 0 & 0 \text { et } 0,035 & 0 \text { et } 0,035 & 0 & 0 & 0 \text { et } 0,035\end{array}$


présence de bismuth [24]. L'adjonction de manganèse destinée à prévenir la formation de $\mathrm{Fe}^{2+}$, n'a pas d'influence sur les résultats obtenus compte tenu de la faible valeur de $m$. Les distributions des cations indiqués par les formules (1), (2) et (3) ont été vérifiées par diffraction de neutrons et de rayons X [9]; aucun écart significatif n'a été observé.

Les mesures sur les ferrites de $\mathrm{Li}-\mathrm{Cr}$ ont été effectuées à partir de poudre de monocristaux préparés par la méthode de flux, le fondant utilisé étant $\mathrm{PbO}-\mathrm{B}_{2} \mathrm{O}_{3}[25]$.

Les mesures par spectrométrie Mössbauer ont été réalisées en utilisant un spectromètre par transmission opérant suivant le mode d'accélération constante. La source utilisée est du $\mathrm{Co}^{57}$ dans une matrice de $\mathrm{Pd}$. La gamme de température étudiée va de $4,2 \mathrm{~K}$ à $300 \mathrm{~K}$.

L'examen des spectres Mössbauer des ferrites de Li-Zn, de Li-Ti et de Li-Zn-Ti pour certains taux de substitution montre une évolution (Figs. 1 et 2 )

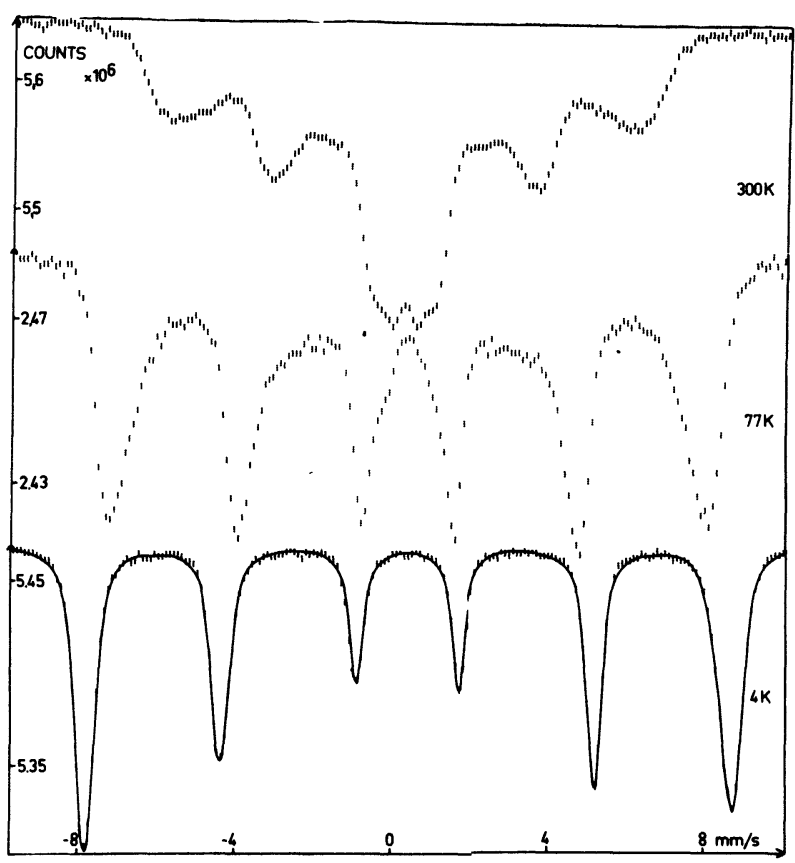

Fig. 1. - Evolution des spectres Mössbauer de $\mathrm{Fe}_{2,2} \mathrm{Li}_{0,2} \mathrm{Zn}_{0,6} \mathrm{O}_{4}$ en fonction de la température. Les traits verticaux correspondent aux points expérimentaux, la ligne pleine au spectre calculé.

[Temperature dependence of Mössbauer spectra of

$$
\mathrm{Fe}_{2.2} \mathrm{Li}_{0.2} \mathrm{Zn}_{0.6} \mathrm{O}_{4} \text {.] }
$$

anormale en fonction de la température : assymétries, élargissements importants, écarts aux intensités théoriques des raies. Ceci est caractéristique des phénomènes de relaxation qui seront explicités et discutés ci-dessous. La limite de la zone de température où apparaissent ces phénomènes est difficile à définir avec précision. Pour $z=0,25$, elle se situe entre $77 \mathrm{~K}$ et $200 \mathrm{~K}$, pour $z=0,6$ elle est proche de $4 \mathrm{~K}$. Pour $t=0,735$, elle se situe entre 100 et $200 \mathrm{~K}$, pour $t \lesssim 0,6$ elle est, si elle existe, supérieure à $300 \mathrm{~K}$.

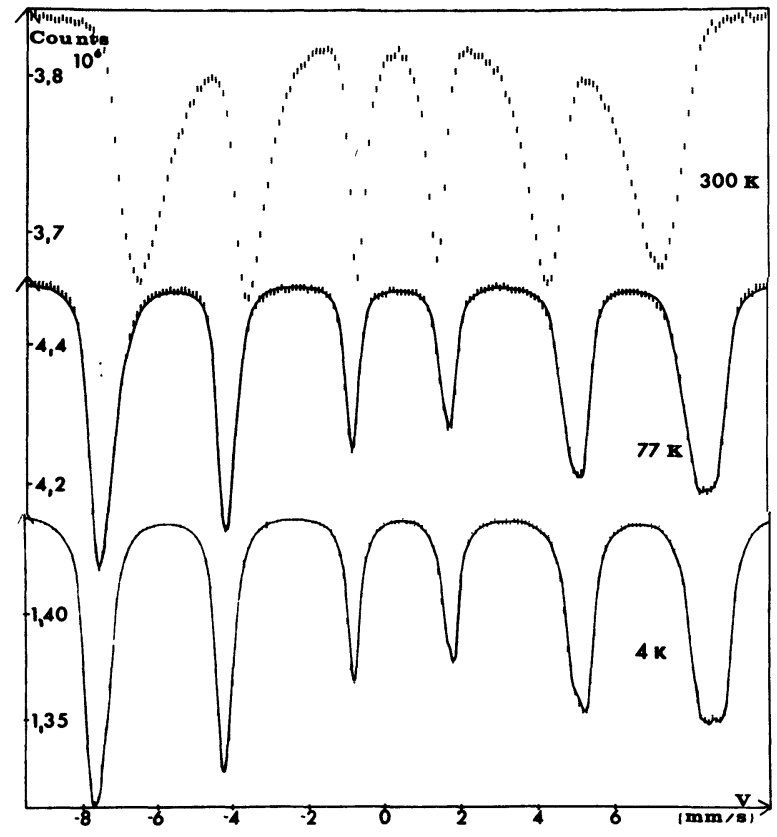

Fig. 2. - Evolution du spectre Mössbauer de $\mathrm{Fe}_{1,4} \mathrm{Li}_{0,865} \mathrm{Ti}_{0,735} \mathrm{O}_{4}$ en fonction de la température. Les traits verticaux correspondent aux points expérimentaux, la ligne pleine au spectre calculé.

[Temperature dependence of Mössbauer spectra of

$$
\mathrm{Fe}_{1.4} \mathrm{Li}_{0.865} \mathrm{Ti}_{0.735} \mathrm{O}_{4} \text {.] }
$$

La détermination des paramètres hyperfins a été faite pour une température située en dehors de la zone de relaxation de façon que celle-ci ne perturbe pas leurs valeurs, soit $T=4 \mathrm{~K}$. Chaque fois que cela a été possible, les spectres des autres températures ont été analysés.

La méthodologie est la suivante : dans une première étape, les spectres ont été analysés sans aucune contrainte, le but étant d'obtenir une bonne reproduction des spectres expérimentaux avec le minimum de sites élémentaires. Cette analyse ne peut être retenue car la solution obtenue ne présente pas de continuité suivant $z, t$ ou $x$ et les valeurs des déplacements isomériques $\delta_{\mathrm{A}}$ et $\delta_{\mathrm{B}}$ sont différentes des valeurs habituellement déterminées pour les ferrites. Dans une deuxième étape, l'influence possible des premiers voisins $\mathrm{Li}$, $\mathrm{Zn}, \mathrm{Ti}, \mathrm{Cr}$ sur les champs hyperfins des $\mathrm{Fe}_{\mathrm{A}}$ et des $\mathrm{Fe}_{\mathrm{B}}$ a été considérée. Les essais des différentes possibilités ont été effectués en imposant les intensités $\alpha$ des spectres élémentaires proportionnels aux probabilités de trouver $0, \ldots, \mathrm{n}$ premiers voisins de la substitution considérée, $\sum \alpha_{A}$ et $\sum \alpha_{B}$ étant déduits des formules de distribution de cation. Pour tester les solutions retenues, les intensités n'ont plus été imposées, soit en gardant $\sum \alpha_{A}$ et $\sum \alpha_{B}$ constants et égaux aux valeurs précédentes, soit sans aucune contrainte.

Une seule solution satisfaisante a été trouvée pour chaque série de ferrites et est résumée dans le tableau II.

Les spectres sont représentés sur les figures 1,2 , 3,4 et 5 et les valeurs des champs hyperfins sont indiquées dans les tableaux III, IV, V et VI. La seule 
Tableau II. - Nombre de sites élémentaires nécessaire à une reproduction correcte des spectres expérimentaux; les raisons de plusieurs sites A ou B sont'indiquées, la largeur de raie est voisine de celle du fer métallique sauf indications contraires.

$$
\mathrm{Li}-\mathrm{Zn}
$$$$
1
$$

Site A

Site B
Plusieurs : influence du $\mathrm{Zn}_{\mathrm{A}}$
$\mathrm{Li}-\mathrm{Zn}-\mathrm{Ti}$

1

(élargi)

Plusieurs : influence du $\mathrm{Zn}_{\mathrm{A}}$
Li-Ti

Plusieurs : influence du $\mathrm{Ti}_{\mathbf{B}}$

Plusieurs : influence du $\mathrm{Li}_{\mathrm{A}}$

\section{$\mathrm{Li}-\mathrm{Cr}$}

1

(élargi)

Plusieurs : influence du $\mathrm{Cr}_{B}$

comparaison possible est la mesure d'Abedilo et al. [15] sur le ferrite de Li-Zn pour $z=0,3$ à $T=4 \mathrm{~K}$. D'après leurs spectres, les différents champs hyperfins se situent entre 510 et $535 \mathrm{kOe}$, ce qui est en accord avec nos mesures.

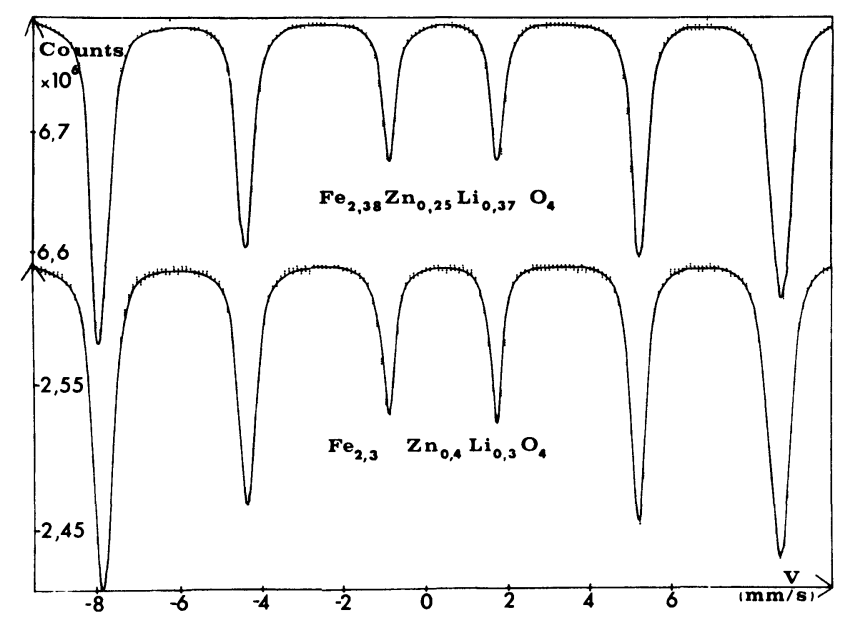

Fig. 3. - Spectres Mössbauer expérimentaux et calculés des ferrites de $\mathrm{Li}-\mathrm{Zn}$ à $T=4 \mathrm{~K}$.

[Mössbauer spectra of $\mathrm{Li}-\mathrm{Zn}$ ferrites at $4 \mathrm{~K}$.]

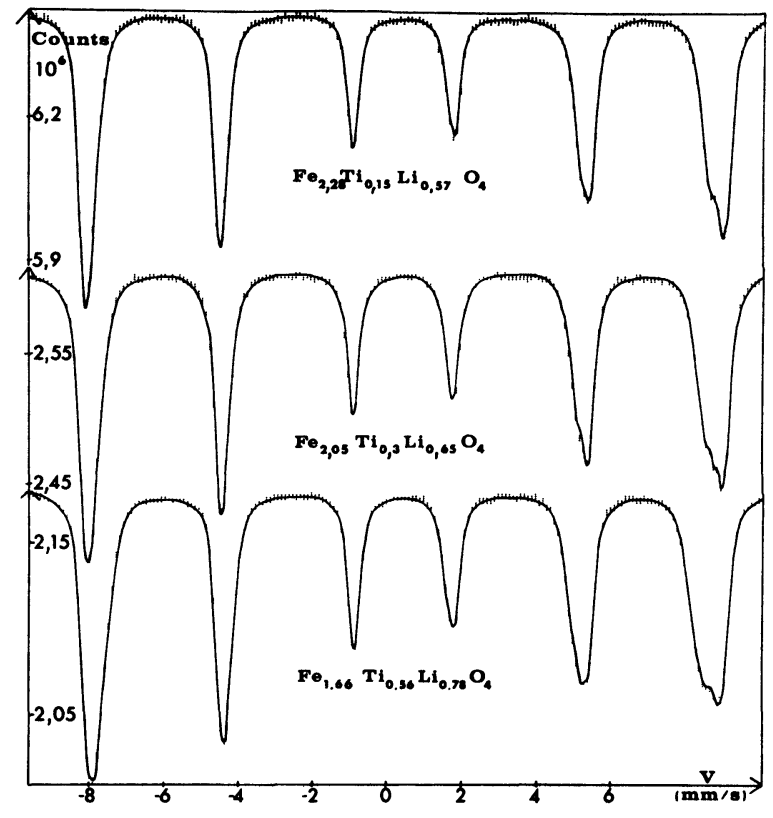

Fig. 4. - Spectres Mössbauer expérimentaux et calculés des ferrites de Li-Tià $T=4 \mathrm{~K}$

[Mössbauer spectra of Li-Ti ferrites at $4 \mathrm{~K}$.]

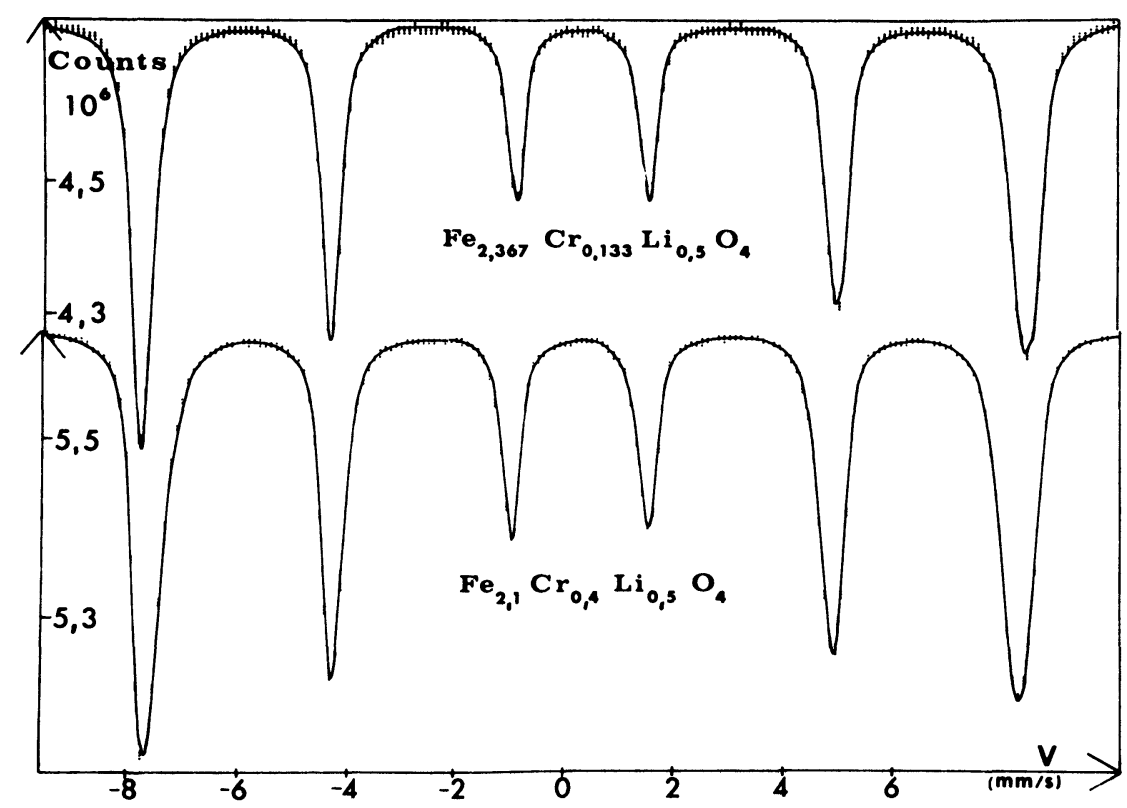

Fig. 5. - Spectres Mössbauer expérimentaux et calculés des ferrites de $\mathrm{Li}-\mathrm{Cr}$ à $T=300 \mathrm{~K}$.

[Mössbauer spectra of Li-Cr ferrites at $300 \mathrm{~K}$.] 
Tableau III. - Champs hyperfins en $\mathrm{kOe} d u \mathrm{Fe}_{\mathrm{A}}$ et des différents sites élémentaires du $\mathrm{Fe}_{\mathrm{B}}$ correspondant à 0 ... 6 proches voisins $\mathrm{Zn}$ en site $\mathrm{A}$, pour différentes compositions et températures des ferrites de $\mathrm{Li}-\mathrm{Zn}$.

$\begin{array}{lccccccccc} & & \mathrm{A} & 0 \mathrm{Zn} & 1 \mathrm{Zn} & 2 \mathrm{Zn} & 3 \mathrm{Zn} & 4 \mathrm{Zn} & 5 \mathrm{Zn} & 6 \mathrm{Zn} \\ z=0,25 & 77 \mathrm{~K} & 517 \pm 1 & 531 \pm 1 & 519 \pm 1 & 505 \pm 2 & 492 \pm 5 & - & - & - \\ z=0,25 & 4 \mathrm{~K} & 523 \pm 1 & 535 \pm 2 & 524 \pm 1 & 512 \pm 2 & 503 \pm 2 & \simeq 490 & - & - \\ z=0,4 & 4 \mathrm{~K} & 519 \pm 1 & \simeq 537 & 526 \pm 2 & 517 \pm 1 & 506 \pm 1 & 497 \pm 2 & \simeq 484 & - \\ z=0,6 & 4 \mathrm{~K} & 518 \pm 1 & - & \simeq 540 & 531 \pm 2 & 519 \pm 1 & 507 \pm 2 & 498 \pm 2 & \simeq 485\end{array}$

Tableau IV. - Champs hyperfins en kOe des différents sites élémentaires du $\mathrm{Fe}_{\mathrm{B}}$ correspondant à $0 \ldots 6$ proches voisins $\mathrm{Li}$ en site $\mathrm{A}$, pour différentes compositions des ferrites de $\mathrm{Li}-\mathrm{Ti} \dot{a} T=4 \mathrm{~K}$.

$\begin{array}{ccccccc}t & 0 \mathrm{Li} & 1 \mathrm{Li} & 2 \mathrm{Li} & 3 \mathrm{Li} & 4 \mathrm{Li} & 5 \mathrm{Li} \\ - & - & - & - & - & - & - \\ 0,15 & 535 \pm 2 & 523 \pm 3 & \simeq 515 & - & - & - \\ 0,30 & 535 \pm 2 & 524 \pm 2 & 515 \pm 3 & 503 \pm 4 & - & - \\ 0,56 & 534 \pm 2 & 524 \pm 2 & 513 \pm 2 & 500 \pm 5 & \simeq 490 & - \\ 0,735 & \simeq 535 & 523 \pm 2 & 514 \pm 2 & 504 \pm 3 & 493 \pm 4 & \cong 485\end{array}$

L'interaction quadrupolaire est toujours faible, $\left|\varepsilon_{\mathrm{m}}\right|<0,05 \mathrm{~mm} / \mathrm{s}$ et il n'est pas observé d'anomalies dans les valeurs des déplacements isomériques (Tableaux VI, VII et VIII). Enfin, la largeur de raie expérimentale des spectres élémentaires (Tableaux VI, VII et VIII) est presque toujours voisine de celle du fer métallique.

2. Discussion. - En premier lieu sera examinée la variation du champ hyperfin au noyau du fer et son

Tableau V. - Champs hyperfins en $\mathrm{kOe}$ des différents sites élémentaires du $\mathrm{Fe}_{\mathrm{A}}$ correspondant à 0 ... 9 proches voisins $\mathrm{Ti}$ en site $\mathrm{B}$, pour différentes compositions des ferrites de $\mathrm{Li}-\mathrm{Ti} \dot{a} \mathrm{~T}=4 \mathrm{~K}$.

$\begin{array}{cccccccc}t & 0 \mathrm{Ti} & 1 \mathrm{Ti} & 2 \mathrm{Ti} & 3 \mathrm{Ti} & 4 \mathrm{Ti} & 5 \mathrm{Ti} & 6 \mathrm{Ti} \\ - & - & - & - & - & - & - & - \\ 0,15 & 517 \pm 2 & 506 \pm 2 & 494 \pm 3 & - & - & - & - \\ 0,30 & 519 \pm 3 & 507 \pm 1 & 495 \pm 2 & 482 \pm 2 & - & - & - \\ 0,56 & \simeq 525 & 513 \pm 2 & 503 \pm 2 & 491 \pm 2 & 478 \pm 3 & \simeq 465 & - \\ 0,735 & - & \simeq 510 & 500 \pm 5 & 489 \pm 2 & 476 \pm 3 & 464 \pm 3 & \simeq 450\end{array}$

Tableau VI. - Valeurs des champs hyperfins pour le $\mathrm{Fe}_{\mathrm{A}}$ et les différents sites élémentaires du $\mathrm{Fe}_{\mathrm{B}}$ correspondant à $(0 \ldots 6) \mathrm{Cr}_{\mathrm{B}}$ premiers voisins; valeurs des déplacements isomériques par rapport au fer métallique pour le $\mathrm{Fe}_{\mathrm{A}}$ et pour le $\mathrm{Fe}_{\mathrm{B}}$ (les deux chiffres donnent la gamme où s'inscrivent les différentes valeurs des spectres élémentaires); largeur de raie expérimentale $\Delta \Gamma_{\mathrm{ex}}$ d'un spectre élémentaire correspondant au $\mathrm{Fe}_{\mathrm{A}}$ ou au $\mathrm{Fe}_{\mathrm{B}}$, pour différentes compositions et températures des ferrites de $\mathrm{Li}-\mathrm{Cr}$.

\begin{tabular}{|c|c|c|c|c|c|c|c|c|c|c|}
\hline & \multicolumn{5}{|c|}{$H(\mathrm{kOe})$} & \multicolumn{2}{|c|}{$\delta(\mathrm{mm} / \mathrm{s})$} & \multicolumn{2}{|c|}{$\Delta \Gamma_{\mathrm{ex}}(\mathrm{mm} / \mathrm{s})$} \\
\hline & & A & $0 \mathrm{Cr}$ & $1 \mathrm{Cr}$ & $2 \mathrm{Cr}$ & $3 \mathrm{Cr}$ & A & B & A & B \\
\hline & & - & - & - & - & - & - & - & - & - \\
\hline$t=0,133$ & $4 \mathrm{~K}$ & $520 \pm 2$ & $535 \pm 3$ & $521 \pm 3$ & 507 & - & $0,32 \pm 0,04$ & $0,49-0,51$ & $0,40 \pm 0,05$ & $0,32 \pm 0,03$ \\
\hline$t=0,400$ & $4 \mathrm{~K}$ & $518 \pm 2$ & $534 \pm 2$ & $521 \pm 2$ & $505 \pm 3$ & $\simeq 487$ & $0,34 \pm 0,03$ & $0,46-0,49$ & $0,45 \pm 0,05$ & $0,33 \pm 0,03$ \\
\hline$t=0,133$ & $300 \mathrm{~K}$ & $500 \pm 2$ & $506 \pm 2$ & $493 \pm 3$ & $\simeq 480$ & - & $0,21 \pm 0,03$ & $0,35-0,38$ & $0,40 \pm 0,05$ & $0,32 \pm 0,03$ \\
\hline$t=0,400$ & $300 \mathrm{~K}$ & $495 \pm 3$ & $506 \pm 2$ & $493 \pm 2$ & $480 \pm 4$ & $\simeq 460$ & $0,21 \pm 0,03$ & $0,36-0,38$ & $0,43 \pm 0,05$ & $0,31 \pm 0,03$ \\
\hline
\end{tabular}

Tableau VII. - Variation $\Delta H_{\mathrm{HST}}$ du champ hyperfin supertransferré par atome de $\mathrm{Zn}_{\mathrm{A}}$, champ hyperfin $H_{6 \mathrm{Zn}}$ calculé du $\mathrm{Fe}_{\mathrm{B}}$ entouré par 6 proches voisins $\mathrm{Zn}_{\mathrm{A}}$, déplacements isomériques $\delta$ du $\mathrm{Fe}_{\mathrm{A}}$ et du $\mathrm{Fe}_{\mathrm{B}}$ par rapport au fer métallique (les deux chiffres donnent la gamme où s'inscrivent les différentes valeurs des spectres élémentaires), largeur de raie expérimentale $\Delta \Gamma_{\mathrm{ex}}$ d'un spectre élémentaire, pour différentes compositions et températures des ferrites de $\mathrm{Li}-\mathrm{Zn}$.

\begin{tabular}{|c|c|c|c|c|c|c|}
\hline & & $\begin{array}{l}\Delta H_{\mathrm{HST}} \\
(\mathrm{kOe})\end{array}$ & $\begin{array}{c}H_{6 \mathrm{Zn}} \\
(\mathrm{kOe})\end{array}$ & $\begin{array}{c}\delta_{\mathrm{A}} \\
(\mathrm{mm} / \mathrm{s})\end{array}$ & $\begin{array}{c}\delta_{\mathrm{B}}(0 \text { à } 6) \\
(\mathrm{mm} / \mathrm{s})\end{array}$ & $\begin{array}{c}\Delta \Gamma_{\mathrm{ex}} \\
(\mathrm{mm} / \mathrm{s})\end{array}$ \\
\hline & & - & - & - & - & - \\
\hline$z=0,25$ & $77 \mathrm{~K}$ & $-13 \pm 2$ & - & $0,35 \pm 0,02$ & $0,44-0,48$ & $0,37 \pm 0,02$ \\
\hline$z=0,25$ & $4 \mathrm{~K}$ & $-11 \pm 2$ & 470 & $0,36 \pm 0,02$ & $0,47-0,50$ & $0,33 \pm 0,02$ \\
\hline$z=0,4$ & $4 \mathrm{~K}$ & $-10 \pm 2$ & 476 & $0,36 \pm 0,02$ & $0,46-0,49$ & $0,34 \pm 0,02$ \\
\hline$z=0,6$ & $4 \mathrm{~K}$ & $-12 \pm 2$ & 486 & $0,37 \pm 0,02$ & $0,47-0,52$ & $0,36 \pm 0,02$ \\
\hline
\end{tabular}


Tableau VIII. - Variation du champ hyperfin supertransferré $\Delta H_{\mathrm{HST}}(\mathrm{B})$ par atome de $\mathrm{Li}, \Delta H_{\mathrm{HST}}(\mathrm{A})$ par atome de $\mathrm{Ti}$; champ hyperfin calculé $H_{0 \mathrm{Li}}(\mathrm{B}) d u \mathrm{Fe}_{\mathrm{B}}$ entouré par 6 proches voisins $\mathrm{Fe}_{\mathrm{A}}, H_{0 \mathrm{Ti}}(\mathrm{A}) d u \mathrm{Fe}_{\mathrm{A}}$ entouré par 9 proches voisins $\mathrm{Fe}_{\mathrm{B}}$ et 3 proches voisins $\mathrm{Li}_{\mathrm{B}}$; déplacements isomériques $\delta$ du $\mathrm{Fe}_{\mathrm{A}}$ et du $\mathrm{Fe}_{\mathrm{B}}$ par rapport au fer métallique (les deux chiffres donnent la gamme où s'inscrivent les différentes valeurs des spectres élémentaires); largeur de raie expérimentale $\Delta \Gamma_{\mathrm{ex}}$ d'un spectre élémentaire, pour différentes compositions des ferrites de Li-Ti $\grave{a} T=4 \mathrm{~K}$.

\begin{tabular}{|c|c|c|c|c|c|c|c|}
\hline$t$ & $\begin{array}{c}\Delta H_{\mathrm{HST}}(\mathrm{B}) \\
(\mathrm{kOe})\end{array}$ & $\begin{array}{c}H_{\mathrm{OLi}}(\mathrm{B}) \\
(\mathrm{kOe})\end{array}$ & $\begin{array}{c}\Delta H_{\mathrm{HST}}(\mathrm{A}) \\
(\mathrm{kOe})\end{array}$ & $\begin{array}{c}H_{0 \mathrm{Ti}}(\mathrm{A}) \\
(\mathrm{kOe})\end{array}$ & $\begin{array}{c}\delta_{\mathrm{B}}(0 \text { à } 6) \\
(\mathrm{mm} / \mathrm{s})\end{array}$ & $\begin{array}{c}\delta_{\mathrm{A}}(0 \text { à } 9) \\
(\mathrm{mm} / \mathrm{s})\end{array}$ & $\begin{array}{c}\Delta \Gamma_{\mathrm{ex}} \\
(\mathrm{mm} / \mathrm{s})\end{array}$ \\
\hline- & - & - & - & - & - & - & - \\
\hline 0,15 & $-11 \pm 2$ & $535 \pm 2$ & $-12 \pm 2$ & $517 \pm 2$ & $0,47-0,49$ & $0,32-0,36$ & $0,34 \pm 0,02$ \\
\hline 0,30 & $-11 \pm 2$ & $535 \pm 2$ & $-12 \pm 2$ & $519 \pm 2$ & $0,47-0,48$ & $0,31-0,35$ & $0,32 \pm 0,02$ \\
\hline 0,56 & $-11 \pm 2$ & $535 \pm 2$ & $-12 \pm 2$ & $526 \pm 3$ & $0,47-0,51$ & $0,28-0,31$ & $0,34 \pm 0,02$ \\
\hline 0,735 & $-10 \pm 2$ & $534 \pm 3$ & $-12 \pm 2$ & $524 \pm 3$ & $0,48-0,50$ & $0,31-0,33$ & $0,34 \pm 0,02$ \\
\hline
\end{tabular}

évolution en fonction de la température, en particulier la contribution du champ hyperfin supertransferré $H_{\text {HST }}$ sera discutée en détail.

Ensuite les résultats seront commentés du point de vue de la relaxation en liaison avec la structure magnétique non colinéaire. Un résumé d'une partie de ces résultats a été fait dans la référence [26].

2.1 Champs hyperfins a $T=4,2 \mathrm{~K}$. - Le champ hyperfin au noyau du fer peut s'inscrire sous la forme [27] :

$$
H=H_{\mathrm{c}}+H_{\mathrm{orb}}+H_{\mathrm{dip}}+H_{\mathrm{cov}}+H_{\mathrm{HST}}
$$

où $H_{\mathrm{c}}$ est le terme de contact; il correspond à l'influence des couches électroniques internes $\mathrm{s}$ et il est proportionnel à $\left\langle S_{\mathbf{z}}\right\rangle$.

$H_{\text {orb }}$ et $H_{\text {dip }}$ correspondent aux contributions orbitale et dipolaire au champ hyperfin.

$H_{\text {cov }}$ dépend du recouvrement des orbitales et du transfert de spin des anions proches voisins; ce terme est en général très faible dans les ferrites.

$H_{\text {HST }}$ est la contribution due au transfert de spin d'un ion métallique à un autre ion métallique via les ligands [23].

La contribution $H_{\mathrm{HST}}(\mathrm{B})$ à $H(\mathrm{~B})$ dépend donc des moments magnétiques des six premiers voisins $\mathrm{A}$ suivant la liaison $\mathrm{Fe}_{\mathrm{A}}-\mathrm{O}-\mathrm{Fe}_{\mathrm{B}} ; H_{\mathrm{HST}}(\mathrm{B})$ est parallèle à l'aimantation $M_{\mathrm{A}}$ du site $\mathrm{A}$, antiparallèle à $M_{\mathrm{B}}$ et s'ajoute donc à $H(\mathrm{~B})$.

La substitution d'un $\mathrm{Fe}_{\mathrm{A}}$ par un atome non magnétique provoque une diminution $\Delta H_{\mathrm{HST}}(B)$ de $H(\mathrm{~B})$. Si les atomes non magnétiques sont répartis au hasard dans le site $\mathrm{A}$, le spectre du $\mathrm{Fe}_{\mathrm{B}}$ sera constitué de la superposition de sept spectres élémentaires de champ hyperfin $H-n \Delta H_{\mathrm{HST}}$, d'intensité relative égale à la probabilité $P$ de trouver $n$ atomes non magnétiques premiers voisins $(0<n<6)$. Il en sera de même pour $H_{\mathrm{HST}}(\mathrm{A})$ et $\Delta H_{\mathrm{HST}}(\mathrm{A})$ (le nombre de premiers voisins $\mathrm{B}$ est alors 12).

Les valeurs obtenues de $\Delta H_{\mathrm{HST}}$ (B) pour les ferrites de Li-Zn (Tableau VII), les ferrites de Li-Zn-Ti (très semblables aux précédentes, car $t$ est faible), les ferrites de Li-Ti (Tableau VIII) correspondent à

$$
\Delta H_{\mathrm{HST}}(\mathrm{B})=11 \pm 2 \mathrm{kOe} .
$$

Cette valeur est très cohérente avec celles habituellement obtenues pour les ferrites [23] et notamment pour des ferrites de Li-Mn et Li-Ga [28]. Le champ hyperfin $H_{6 \mathrm{Zn}}$ correspondant à un $\mathrm{Fe}_{\mathrm{B}}$ entouré de six voisins $\mathrm{Zn}$ a été déterminé, ce qui permet de déduire $H_{6 \mathrm{Zn}}=505 \pm 7 \mathrm{kOe}$ pour $z=1$ en bon accord avec la valeur obtenue pour $\mathrm{ZnFe}_{2} \mathrm{O}_{4}$ (512 kOe [29]). De même, la valeur de $H_{0 z n}(B)$ (ferrites de Li-Zn) et la valeur de $H_{0 \mathrm{Li}}(\mathrm{B})$ (ferrites de Li-Ti) (Tableau VIII) ont été déterminées ce qui permet de calculer $H_{0 \mathrm{Zn}}(\mathrm{B})=530 \pm 4 \mathrm{kOe}$ pour $z=0 \mathrm{et}$

$$
H_{0 \mathrm{Li}}(\mathrm{B})=535 \pm 2 \mathrm{kOe}
$$

pour $t=0$, valeurs en bon accord avec celle obtenue pour le ferrite de $\mathrm{Li}$ pur [30] $\left(\bar{H}_{\mathrm{B}}=537 \pm 2 \mathrm{kOe}\right)$.

Etant trop faible ( $\cong 3 \mathrm{kOe}$ ) la valeur habituellement admise pour $H_{\mathrm{HST}}(\mathrm{A})$ [31-32] ne permet pas d'observer une distribution des sites A. Ceci est confirmé par les mesures sur les ferrites de $\mathrm{Li}-\mathrm{Zn}$ et sur le ferrite de Li pur [30]. Il n'en est pas de même pour les ferrites de $\mathrm{Li}-\mathrm{Ti}$ où une distribution des sites $\mathrm{A}$ a été considérée pour obtenir une bonne reproduction des spectres. Les intensités des spectres élémentaires montrent que seul le $\mathrm{Ti}$ a une influence sur $H_{\mathrm{HST}}(\mathrm{A})$ et qu'il peut occuper neuf des douze premiers voisins. Ceci indiquerait que le $\mathrm{Li}$ reste quasi ordonné (le $\mathrm{Li}$ occupe en moyenne $1 / 4$ des sites B comme dans le ferrite de $\mathrm{Li}$ pur). La valeur obtenue de $\Delta H_{\mathrm{HST}}(\mathrm{A})$ est de $12 \pm 2 \mathrm{kOe}$ (Tableau VIII) et pourrait être due à un effet de taille, l'ion $\mathrm{Ti}$ étant relativement gros. La valeur déduite $H_{0 \mathrm{Ti}}(\mathrm{A})=516 \pm 3 \mathrm{kOe}$ (Tableau VIII) pour $t=0$, la valeur de $H_{\mathrm{A}}=525 \pm 3 \mathrm{kOe}$ (Tableau III) pour $z=0$, la valeur de

$$
H_{\mathrm{A}}=522 \pm 2 \mathrm{kOe}
$$

(Tableau VI) pour $x=0$ sont en bon accord avec la valeur de $\bar{H}_{\mathrm{A}}=520 \pm 2 \mathrm{kOe}$ mesurée pour le ferrite de Li pur [30].

Pour les ferrites de $\mathrm{Li}-\mathrm{Zn}-\mathrm{Ti}$ et de $\mathrm{Li}-\mathrm{Cr}$, seul un élargissement du spectre élémentaire du site $\mathrm{A}$ est observé. Il serait dû à l'effet de la substitution de Ti (faible, $t=0,15$ ) dans le premier cas. Pour les ferrites de $\mathrm{Li}-\mathrm{Cr}$, la raison peut être double. D'une part, le $\mathrm{Cr}^{3+}$ n'a pas le même moment magnétique que le 
$\mathrm{Fe}^{3+}$ et il en résulte un $\Delta H_{\mathrm{HST}}(\mathrm{A})$, d'autre part, la substitution de $\mathrm{Cr}$ détruit l'ordre du Li [25] ce qui provoque un autre $\Delta H_{\mathrm{HST}}(\mathrm{A})$.

$H_{\mathrm{HST}}(\mathrm{B})$ étant constant pour les ferrites de $\mathrm{Li}-\mathrm{Cr}$, la variation de $H(\mathrm{~B})$ ne peut être due qu'à une variation de $H_{\text {dip }}$ ou une variation de $\left\langle S_{z}\right\rangle$. La valeur de $H$ (B) observée, égale à $-15 \pm 2 \mathrm{kOe}$ (Tableau VI) par atome de $\mathrm{Cr}$ substituant le $\mathrm{Fe}$, semble trop grande pour être interprétée par une variation de $H_{\text {dip }}$. De plus, celle-ci doit dépendre du nombre d'atomes substitués, mais aussi de leur position, ce qui ne semble pas le cas. La variation locale de $\left\langle S_{z}\right\rangle$ semble plus raisonnable car le moment magnétique du $\mathrm{Cr}$ est d'environ $1 \mu_{\mathrm{B}}$, plus faible que celui du Fe et le rapport des intégrales d'échanges $J_{\mathrm{BB}} / J_{\mathrm{AB}}$ est plus grand pour le $\mathrm{Cr}$, ce qui renforce la tendance antiferromagnétique à l'intérieur du site $B$.

Il est à noter que la structure non colinéaire des $\mathrm{Fe}_{\mathbf{B}}$ dans les ferrites de $\mathrm{Li}-\mathrm{Zn}$ a une influence sur les $\Delta H_{\mathrm{HST}}(\mathrm{B})$ déterminé :

$$
H_{n}(\mathrm{~B})=H_{6 \mathrm{Zn}}+n \Delta H_{\mathrm{HST}}(\mathrm{B}) \times \cos \theta_{n}
$$

où $n$ est le nombre de premiers voisins Fe et $\theta_{n}$ l'angle entre $M_{\mathrm{B}}$ et $M_{\mathrm{A}}$. En prenant les valeurs de $\theta_{n}$ déterminées pour les ferrites de $\mathrm{Ni}-\mathrm{Zn}$ [19-20], la différence entre $H_{n}$ et $H_{n-1}$ n'excède pas la valeur moyenne de $\Delta H_{\mathrm{HST}}(\mathrm{B})$ de $\pm 2 \mathrm{kOe}$ pour $n \geqslant 1$ : le phénomène n'est donc pas observable.

\subsection{VARIATION DES CHAMPS HYPERFINS EN FONCTION} DE LA TEMPÉRATURE. - Dans la zone de température étudiée, la variation des champs hyperfins des ferrites de $\mathrm{Li}-\mathrm{Cr}$ ne présente pas de particularité. Pour les ferrites de $\mathrm{Li}-\mathrm{Ti}$, la solution employée pour $T=4 \mathrm{~K}$ permet d'obtenir de bonnes reproductions des spectres expérimentaux, quoique le facteur $" \chi^{2}$ » augmente à $T=300 \mathrm{~K}$ (surtout pour $t=0,56$ ). Lorsque les intensités des spectres élémentaires sont laissées libres, des solutions incohérentes sont souvent obtenues (sans amélioration notable du facteur $\left." \chi^{2} »\right)$, mais ceci peut provenir d'une part des phénomènes de relaxation qui commence à perturber les spectres, d'autre part des valeurs des déplacements isomériques $\delta_{\mathrm{A}}$ et $\delta_{\mathrm{B}}$ qui deviennent plus proches et qui permettent de moins en moins de séparer les sites élémentaires A des $\mathrm{B}$. En particulier à $T=300 \mathrm{~K}$,

$\bar{\delta}_{\mathrm{A}}=0,28 \pm 0,03 \mathrm{~mm} / \mathrm{s}$ et $\bar{\delta}_{\mathrm{B}}=0,34 \pm 0,03 \mathrm{~mm} / \mathrm{s}$

alors qu'à $T=4 \mathrm{~K}, \quad \bar{\delta}_{\mathrm{A}}=0,37 \pm 0,03 \mathrm{~mm} / \mathrm{s}$ et $\bar{\delta}_{\mathrm{B}}=0,50 \pm 0,03 \mathrm{~mm}$ correspondant à une variation de $-5,3 \times 10^{-4} \mathrm{~mm} / \mathrm{s} / \mathrm{K}$ pour le site $B$ et de $-3 \times 10^{-4} \mathrm{~mm} / \mathrm{s} / \mathrm{K}$ pour le site $A$. Ces valeurs sont identiques à celles obtenues pour le ferrite de $\mathrm{Li}$ pur [30]. Les variations du champ hyperfin d'un spectre élémentaire correspondant à $\mathrm{n} \mathrm{Li}$ ou $\mathrm{Ti}$ premiers voisins normalisé à sa valeur à $T=4 \mathrm{~K}$ en fonction de $T / T_{\mathrm{c}}$ ont été tracées sur la figure 6. Pour $n=0$ à $3 \mathrm{Li}$ et $n=0$ à $2 \mathrm{Ti}$, les courbes se confondent, pour $n=3$ et $4 \mathrm{Ti}$, la variation est plus rapide, quoique

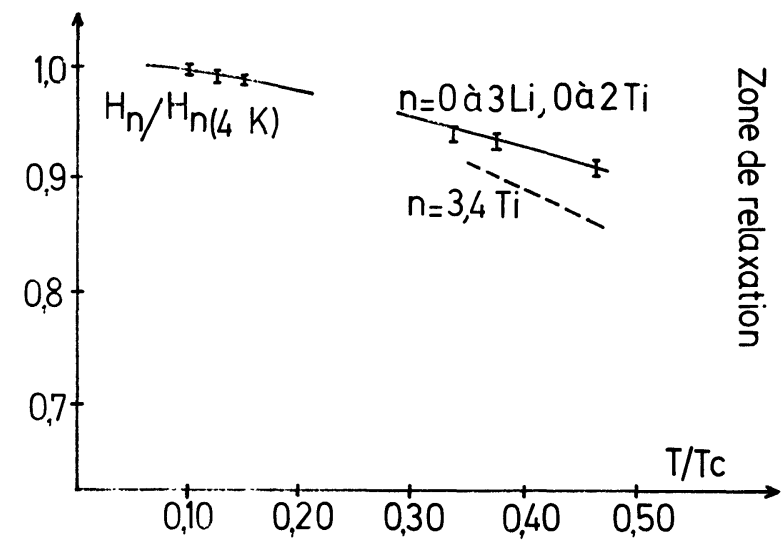

Fig. 6. - Variation du champ hyperfin correspondant à un site élémentaire de $\mathrm{Fe}$ ayant $n \mathrm{Li}$ ou Ti premiers voisins normalisé à sa valeur à $4 \mathrm{~K}$ en fonction de $T / T_{\mathrm{c}}$.

$\left[T / T_{\mathrm{c}}\right.$ dependence of hyperfine field of an elementary site of $\mathrm{Fe}$ having $n \mathrm{Li}$ or Ti as first neighbours normalised to its value at $4 \mathrm{~K}$.]

deux des points expérimentaux sur trois correspondent à la substitution $t=0,56$ pour $T=300 \mathrm{~K}$ où l'influence de la relaxation est probable (au début, celle-ci élargie assymétriquement les spectres et peut être simulée par des spectres élémentaires ayant des champs hyperfins plus faibles). Les valeurs obtenues sont de toute façon nettement moins différenciées que les valeurs prévues; par exemple, pour le site B ( $n$ alors varie de 0 à 6$)$ et $T / T_{\mathrm{c}}=0,4, H_{n} / H_{n 4 \mathrm{~K}}$ varie entre $0,94(n=0$ et 1$)$ et $0,84(n=3)$ [16], alors que $0,93 \pm 0,01$ est obtenu d'après nos mesures. Pour les ferrites de $\mathrm{Li}-\mathrm{Zn}$, seul le spectre à $T=77 \mathrm{~K}$ pour $z=0,25$ n'est pas affecté par la relaxation quoique pour cette composition, le facteur " $\chi^{2}$ " augmente ainsi que la largeur de raie (Tableau VII); les valeurs obtenues sont cohérentes avec celles de $T=4 \mathrm{~K}$, avec un $\Delta H_{\mathrm{HST}}$ légèrement plus grand.

\subsection{RELAXATION ET STRUCTURE NON COLINÉAIRE} LOCALE. - Dès que la température est différente de zéro, les spins nucléaires sont dans un environnement fluctuant. Ces fluctuations peuvent concerner directement le spin nucléaire, ou les niveaux électroniques de l'atome par exemple par couplage entre les niveaux et le réseau, entre les niveaux de deux atomes...; dans ce dernier cas, les fluctuations sont transmises au spin nucléaire par l'intermédiaire de l'interaction entre le noyau et les couches électroniques de l'atome. L'effet de cette relaxation va dépendre de sa période $\tau$ par rapport au temps de mesure. Pour la spectrométrie Mössbauer, ce temps est égal à $\tau_{\mathrm{e}}$, durée de vie de l'état excité, soit $\tau_{\mathrm{e}} \simeq 2 \times 10^{-8} \mathrm{~s}$ pour le ${ }^{57} \mathrm{Fe}$. Si $\tau \gg \tau_{e}$, la relaxation n'a pas d'effet et les paramètres ne sont pas perturbés; si $\tau \ll \tau_{\text {e }}$, les paramètres mesurés seront égaux à la moyenne dans le temps des paramètres fluctuants. $\mathrm{Si} \tau \simeq \tau_{\mathrm{e}}$, la situation est intermédiaire et les spectres Mössbauer présentent des déformations. Pour traiter de ce cas, des modèles ont été développés à partir de la théorie stochastique [33] 
ou du traitement ab initio [34]. Ces modèles rendent bien compte de l'évolution de la forme des spectres en fonction de $\tau$ (et de la température $T$ puisque $\tau$ diminue lorsque $T$ augmente).

Les spectres des ferrites substitués avec du $\mathrm{Zn}$ présentent effectivement une évolution anormale en fonction de la température. Un premier essai de reproduction a été fait à partir de superposition de spectres élémentaires ferromagnétiques et paramagnétiques [14], mais le résultat est médiocre, ce qui rend peu vraisemblable l'hypothèse de fer devant paramagnétique. Une autre possibilité évoquée a été celle d'une transition ferromagnétique-superparamagnétique, mais celle-ci ne peut être retenue car son évolution en température est différente. En effet, si l'on considère un ensemble de grains, particules, ... de volumes semblables, donc d'énergies de barrière semblables, la transition a lieu dans un domaine de températures assez étroit. S'il y a distribution des volumes, le domaine est plus large mais on observe alors la superposition de spectres élémentaires ferromagnétiques déformés et superparamagnétiques (dans ce cas, des singulets puisque $\varepsilon_{\mathrm{m}} \simeq 0$ ); l'application d'un champ magnétique même faible provoque le retour à l'état ferromagnétique. Or l'ensemble de ces propriétés n'est pas observé [16, 21, 22]. Par contre, en utilisant les théories développées à partir du modèle stochastique, Bhargava et al. [21, 22] ont bien reproduit l'évolution en température des spectres. Mais ils n'ont ni tenu compte de la structure non colinéaire (qui intervient dans les calculs par le biais du champ moléculaire) ni des $H_{\text {HST }}$ différents. Ceci ne met pas en cause la validité de leurs travaux, mais doit modifier les valeurs des $\tau$ obtenus. Pour refaire ce calcul dans les meilleures conditions, il manque néanmoins une donnée. En effet, la pente plus faible de l'aimantation en fonction du champ appliqué à hautes températures, les mesures de Zhilyakov et al. [10], vont plutôt dans le sens d'une diminution des angles locaux $\theta$ entre $\mathbf{S}_{\mathbf{A}}$ et $\mathbf{S}_{\mathbf{B}}$ lorsque la température augmente, mais cette variation reste à confirmer.

Les mesures effectuées sur les ferrites de $\mathrm{Li}-\mathrm{Zn}$ donnent des résultats très semblables à ceux obtenus sur les ferrites substitués avec du $\mathrm{Zn}$. Mais des phénomènes de relaxation sont aussi présents pour les ferrites de $\mathrm{Li}-\mathrm{Ti}$ : notons la similitude de forme entre le spectre du Li-Zn à $T=77 \mathrm{~K}$ pour $z=0,6$ (Fig. 1) et le spectre du Li-Ti à $T=300 \mathrm{~K}$ pour $t=0,735$ (Fig. 2). Par rapport aux ferrites de Li-Zn ces phénomènes se situent pour des taux de substitution et des températures plus élevées, ce qui indique que les temps de relaxation sont plus grands dans les ferrites de Li-Ti.

Plusieurs tentatives ont été effectuées pour tenter d'expliquer les phénomènes de relaxation. L'hypothèse basée sur la relaxation des parois des domaines magnétiques [35] peut être écartée car l'application d'un champ magnétique ferait disparaître ces parois, donc la relaxation. Mais est-ce une relaxation spin- réseau [16] ou une relaxation spin-spin due au manque de corrélation à longue distance [21], ou est-ce lié à la structure non colinéaire (non contradictoire avec l'hypothèse précédente). L'application d'un champ magnétique n'apporte pas beaucoup d'informations supplémentaires car s'il fait diminuer l'angle local $\theta$, il modifie aussi les temps de relaxation, ce qui affecte dans le même sens l'évolution des spectres.

Si effectivement la relaxation et la structure non colinéaire de $\mathbf{S}_{\mathbf{A}}$ et $\mathbf{S}_{\mathbf{B}}$ sont liées, les ferrites de Li-Ti doivent présenter aussi une structure non colinéaire locale du même type : ce serait alors $S_{A}$ qui ferait un angle local $\theta$ avec $\mathbf{S}_{\mathrm{B}}$ de par la substitution de Ti en site B. Ceci d'ailleurs permettrait d'expliquer la variation de l'aimantation à $0 \mathrm{~K}$ pour $t \geqq 0,7$; l'angle moyen $\bar{\theta}$ peut être évalué à environ $\bar{\theta} \simeq 10^{\circ}$ pour $t=0,8^{\prime}, \bar{\theta} \simeq 20^{\circ}$ pour $t=1,0$ et $\bar{\theta} \simeq 35^{\circ}$ pour $t=1,2$. Les résultats préliminaires de mesures d'aimantation à forts champs appliqués (jusqu'à $150 \mathrm{kOe}$ ) sur les compositions $t=0,735,1,9$ et 1,1 confirment la présence d'une structure non colinéaire. Pour les ferrites de Li-Ti, $\bar{\theta}$ est nettement plus faible que pour les ferrites de Li-Zn. Ceci est cohérent avec le L.C.M. car $\theta$ dépend du rapport des intégrales d'échange $J_{\mathrm{BB}} / J_{\mathrm{AB}}$ pour le site B et $J_{\mathrm{AA}} / J_{\mathrm{AB}}$ pour le site A et $J_{\mathrm{AA}}<J_{\mathrm{BB}}$. Enfin, on peut remarquer que les phénomènes de relaxation sont observables $(\tau \simeq \tau$ de mesure) que si il existe une structure non colinéaire et que le temps de relaxation $\tau$ diminue lorsque $\bar{\theta}$ augmente, ce qui semble bien lier les deux phénomènes.

Dernière remarque, il a été constaté que le champ hyperfin $H_{n} / H_{n 4 \mathrm{~K}}$ (chapitre précédent) d'un fer entouré par $n$ premiers voisins non magnétiques diminue avec la température d'autant plus vite que $n$ est grand. Nous avons montré que ceci n'était pas exact pour les ferrites de $\mathrm{Li}$-Ti lorsque seulement les mesures en dehors de la zone de relaxation sont considérées. Cette diminution de $H_{n} / H_{n 4 \mathrm{~K}}$ n'est-elle pas une simulation de phénomènes de relaxation en début de zone ? La relaxation et la structure non colinéaire seraient alors des phénomènes toujours présents dans les ferrites substitués avec des atomes non magnétiques.

3. Conclusion. - L'étude de ferrites de Li substitués a permis d'apporter des données supplémentaires sur les champs hyperfins supertransferrés $H_{\mathrm{HST}}$ en particulier pour les ferrites de Li-Ti où $H_{\mathrm{HST}}(\mathrm{A})$ est plus grand que les valeurs usuelles. Comme pour les ferrites de $\mathrm{Li}-\mathrm{Zn}$, les phénomènes de relaxation sont présents pour les ferrites de $\mathrm{Li}-\mathrm{Ti}$, quoique pour des températures et des taux de substitution plus élevés. Ces phénomènes pourraient être une règle générale lorsque des atomes non magnétiques sont substitués au fer, ce qui nécessiterait alors un réexamen des valeurs connues des variations de $H_{\mathrm{HST}}$ en fonction de la température, la relaxation pouvant perturber sérieusement leur détermination. Enfin, ces phéno- 
mènes de relaxation sont reliés à l'existence d'une structure non colinéaire locale pour les spins d'un des deux sous-réseaux du fer ( $\mathrm{Fe}_{\mathrm{B}}$ pour les ferrites de $\mathrm{Li}-\mathrm{Zn}, \mathrm{Fe}_{\mathrm{A}}$ pour les ferrites de $\mathrm{Li}-\mathrm{Ti}$ ). Dans ce cas, le manque de corrélation à longue distance des spins dû à cette structure pourrait être la cause de la relaxation.
Remerciements. - Nous remercions vivement MM. R. Krishnan et W. Simonet pour nous avoir aimablement fourni les matériaux, MM. Noguès et P. Gibart pour les discussions qui nous ont beaucoup aidé dans ce travail et M. P. Renaudin pour l'aide technique qu'il a apportée.

\section{Bibliographie}

[1] Blasse, G., Philips Res. Rep. 3 (1964) 1.

[2] Gorter, E. W., Philips Res. Rep. 9 (1954) 295.

[3] West, R. G. et Blankenship, A. C., J. Am. Ceram. Soc. 50 (1967) 343.

[4] Yafet, Y. et Kittel, C., Phys. Rev. 87 (1952) 290.

[5] Geller, S., J. Appl. Phys. 37 (1966) 1408.

[6] Néel, L., C.R. Hebd. Séan. Acad. Sci. 230 (1950) 375.

[7] Gilleo, M. A., J. Phys. Chem. Solids 13 (1960) 33.

[8] Nowik, I., Phys. Rev. 171 (1968) 550

[9] Noguès, M., Dormann, J. L., Perrin, M., Simonet, W. et Gibart, P., I.E.E.E. Trans. on Magn. MAG 15 (1979) 1729.

[10] Zhilyakov, S. M., Ivolga, V. V., Mal'tsev, V. I. et Naiden, E. P., Sov. Phys. Solid State 19 (1977) 1817, Pis'ma Zn. Eksp. Teor. Fiz. 26 (1977) 711.

[11] Rosencwaig, A., Can. J. Phys. 48(1970) 2857.

[12] White, G. O., Edmonson, C. A., Goldfarb, R. B. et PatTON, C. E., J. Appl. Phys. 50 (1979) 2381.

[13] White, G. O. et Patton, C. E., J. Magn. Magn. Mat. 9 (1978) 299.

[14] Young, J. W. et Smith, J., J. Appl. Phys. 42 (1971) 2344.

[15] Abelido, C. R. et Frankel, R. B., J. Physique Paris 38 (1977) $\mathrm{Cl}-135$.

[16] Daniels, J. M. et Rosencwaig, A., Can.J. Phys. 48 (1970) 381.

[17] Leung, L. K., Evans, B. J. et Morrish, A. H., Phys. Rev. 8 (1973) 29.

[18] Clark, P. E. et Morrish, A. H., Phys. Status Solidi A 19 (1973) 687.

[19] Petitt, G. A., Solid. State Commun. 13 (1973) 1611.
[20] Piekoszewski, J., Dabrowski, L. et Suwalski, J., Solid State Commun. 16 (1975) 75, Acta Phys. Pol. A 51 (1977) 179.

[21] Bhargava, S. C. et Iyengar, P. K., Phys. Status Solidi B 53 (1972) 359, J. Physique Colloq. 35 (1974) C6-669.

[22] Bhargava, S. C., Morup, S. et Knudsen, J. E., J. Physique Colloq. 37 (1976) C6-93.

[23] Van der Woude, F. et Sawatzky, I. A., Phys. Rev. B 4 (1971) 3159.

[24] Simonet, W. et Hermosin, A., IEEE Trans. on Magn. 14 (1978) 903.

[25] Krishnan, R., Physica 86-88B (1977) 1457.

[26] Dormann, J. L., J. Physique Colloq. 41 (1980) C1-175.

[27] Consulter par exemple le chapitre 2 de Hyperfine Interactions, édité par A. J. Freeman et R. B. Frankel (Academic Press) (1967).

[28] Bashkirov, Sh., Iskhahov, R. A., Liberman, A. B., ManenKova, L. K. et SinYa vskiI, V., Izv. Vys. Uchev. Zaved. Fiz. 12 (1975) 89, Sov. Jour. Phys.

[29] Varret, F., Gerard, A. et Imbert, P., Phys. Status Solidi 43 (1971) 723.

[30] Dormann, J. L., Tomas, A. et Noguès, M. (non publié).

[31] Streever, R. L., Phys. Rev. 186 (1969) 285.

[32] Doroshev, V. D., Kovtun, N. M. et Selezner, V. N., Phys. Status Solidi 13 (1972) K41.

[33] Clauser, M. J. et Blume, M., Phys. Rev. B 3 (1971) 583.

[34] Gonzalez-Jimenez, F., Imbert, P. et HaRTMANN-BOutron, F., Phys. Rev. 9 (1974) B95.

[35] Srivastava, C. M., Shringi, S. N. et Bommanavar, A. S., J. Physique Colloq. 38 (1977) Cl-43. 